\title{
Ressecção de bolsa hiperfuncionante para tratamento de hipotonia ocular crônica - Relato de casos
}

\author{
Resection of overfiltration bleb for the treatment of chronic ocular hypotony - \\ Case reports
}

SebastiãoCronemberger ${ }^{1}$ Daniel Vítor de Vasconcelos Santos ${ }^{2}$

Ana Cláudia Monteiro Oliveira ${ }^{3}$

Heloísa Andrade Maestrini ${ }^{3}$

Nassim Calixto ${ }^{1}$
Universidade Federal de Minas Gerais - Serviço de Glaucoma do Hospital São Gerado - Hospital das Clínicas, Av. Professor Alfredo Balena, 110 CEP 30130100 Belo Horizonte (MG)

${ }^{1}$ Professor Titular de Oftalmologia da Faculdade de Medicina da Universidade Federal de Minas Gerais (UFMG).

Residente do $3^{0}$ ano em Oftalmologia do Hospital São Geraldo - Hospital das Clínicas da Universidade Federal de Minas Gerais (UFMG).

${ }^{3}$ Fellow do Serviço de Glaucoma do Hospital São Geraldo - Hospital das Clínicas da Universidade Federal de Minas Gerais (UFMG).

Endereço para correspondência: Rua Martim de Carvalho, 410/501 - Belo Horizonte (MG) CEP 30190-090 E-mail: cronem@task.com.br

Recebido para publicação em 17.09.2003

Versão revisada recebida em 03.02.2004

Aprovação em 06.02.2004

Os autores não têm interesse comercial nos produtos e equipamentos utilizados neste estudo.

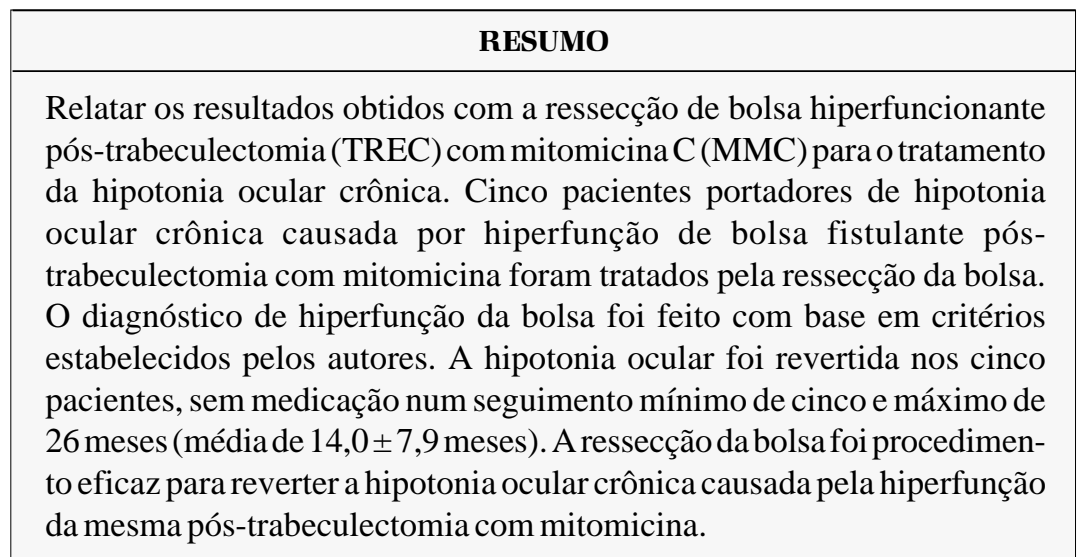

Descritores: Hipotensão ocular; Trabeculectomia; Glaucoma; Mitomicina/uso terapêutico; Injeções; Pressão intra-ocular; Complicações pós-operatórias. Resultado de tratamento; Relato de caso

\section{INTRODUÇ̃̃OO}

Em muitos pacientes glaucomatosos em que há indicação de cirurgia, a trabeculectomia (TREC) tem-se mostrado comprovadamente eficaz na redução da pressão intra-ocular (Po) e na contenção da progressão do dano glaucomatoso ${ }^{(1-4)}$. Essa técnica cirúrgica pode proporcionar benefícios adicionais, tais como a melhora da qualidade de vida do paciente pela redução ou mesmo a abolição do uso de medicação hipotensora ocular e até mesmo a possibilidade de se atingirem Po mais baixas e seguras, principalmente naqueles pacientes em que a doença glaucomatosa encontra-se em estado mais avançado ${ }^{(1)}$. Entretanto, pelo fato dos resultados da TREC não serem sempre satisfatórios, despontaram as estratégias complementares de melhora do funcionamento da fístula, como o uso de antimetabólitos. Em contrapartida, a utilização de antimitóticos trouxe potenciais complicações, entre as quais destaca-se a hipotonia ocular pós-operatória, que pode estar associada à câmara anterior rasa ou mesmo ausente, efusão uveal, catarata e maculopatia ${ }^{(5)}$.

O diagnóstico de certeza assim como o tratamento ideal da bolsa hiperfuncionante ("hiperfiltrante") ainda não estão completamente estabelecidos. Técnicas como a injeção de sangue autólogo na bolsa ${ }^{(6-10)}$, enxerto conjuntival $^{(11-12)}$, enxerto de fáscia lata ${ }^{(13)}$ ou de membrana amniótica ${ }^{(14)}$ e laser $\mathrm{Nd}: \mathrm{YAG}^{(15-16)}$, sutura de compressão ${ }^{(17)}$, uso de lente de contato de grande diâmetro e facoemulsificação ${ }^{(18)}$ têm sido descritas, com eficácia variável, atestando a indefinição de um consenso a esse respeito.

A abordagem cirúrgica propriamente dita da bolsa hiperfuncionante re- 
monta à década de sessenta ${ }^{(19-20)}$ e tem sido crescentemente valorizada na literatura internacional ${ }^{(21-24)}$.

$\mathrm{Na}$ literatura nacional, existem apenas três trabalhos relatando os resultados do tratamento de bolsa hiperfuncionante. Os dois primeiros ${ }^{(14,16)}$ com seguimento pós-operatório muito curto (dois meses e 40 dias respectivamente) envolvem dois pacientes, o primeiro utilizando membrana amniótica ${ }^{(14)}$ e o segundo utilizando sutura de compressão ${ }^{(16)}$. No terceiro trabalho $^{(17)}$, realizado no exterior e publicado no Brasil, são descritos os achados clínicos e as modalidades de tratamento da hipotonia ocular crônica em nove olhos submetidos a TREC primária com mitomicina $\mathrm{C}$ (MMC). Esses três trabalhos não caracterizaram de forma clara quais foram os critérios diagnósticos de hiperfunção ("hiperfiltração") da bolsa.

Dessa forma, o presente estudo tem como objetivo relatar os resultados obtidos com a ressecção de bolsa hiperfuncionante pós-trabeculectomia com mitomicina $\mathrm{C}$ para o tratamento da hipotonia ocular crônica com base em critérios estabelecidos pelo autores para o diagnóstico de bolsa hiperfuncionante.

\section{RELATOS DOS CASOS}

Cinco olhos de cinco pacientes portadores de hipotonia ocular crônica causada por bolsa hiperfuncionante pós-TREC com MMC foram submetidos à ressecção da bolsa.

Foram registrados, de cada paciente, além da idade, cor e sexo: a melhor acuidade visual corrigida do olho com hipotonia, a Po à aplanação, as cirurgias prévias, o aspecto biomicroscópico da bolsa, o resultado do teste de Seidel, os achados oftalmoscópicos e as medicações utilizadas no pré e pósoperatório. Todos os olhos hipotônicos foram também submetidos no pré-operatório a exame de biomicroscopia ultrasônica (UBM).

O diagnóstico de hiperfunção da bolsa fistulante foi feito de maneira criteriosa com base nos seguintes elementos: 1 . Po à tonometria de aplanação inferior a $6 \mathrm{mmHg}$; 2. ausência de sinais inflamatórios no segmento anterior à biomicroscopia; 3 . bolsa fistulante elevada e/ou difusa e avascular com ou sem microcistos no seu interior; 4. ausência de vazamento de humor aquoso ao teste de Seidel, mesmo após leve compressão do globo ocular (teste de Seidel negativo); 5. ausência de descolamento ciliocoroidiano (DCC) à UBM realizada nos quadrantes superior, inferior, nasal e temporal.

Preenchidos os critérios diagnósticos de hiperfunção da bolsa, tentou-se inicialmente normalizar a Po com tratamento clínico (atropina colírio a 1\% uma gota de 12 em 12 horas e colírio de dexametasona a $0,1 \%$ ou similar, uma gota de 6 em 6 horas durante pelo menos trinta dias $)^{(25)}$. Não tendo havido normalização da Po com o tratamento, os pacientes foram submetidos, sob consentimento informado, à ressecção da bolsa hiperfuncionante pelo mesmo cirurgião (SC).

A técnica de ressecção da bolsa foi simples, iniciando-se o ato cirúrgico com a anestesia peribulbar utilizando-se lidocaína a $2 \%(2 \mathrm{ml})$ e bupivacaína a $0,75 \%(6 \mathrm{ml})$ associadas a $0,1 \mathrm{ml}$ de hialuronidase. Em seguida, procedeu-se, à ressecção completa da bolsa fazendo-se uma incisão com uma lâmina de bisturi número 15 ao redor do perímetro de junção entre a bolsa avascular (hiperfuncionante) e a conjuntiva normal. A seguir, procedeu-se à divulsão da conjuntiva normal com tesoura de ponta romba, em direção ao fórnice e, finalmente, efetuou-se a sua mobilização em direção ao limbo onde a conjuntiva foi ancorada com pontos separados de nylon monofilamentar 10-0, sepultando-se o nó da sutura na córnea. $\mathrm{O}$ paciente era liberado após a colocação de pomada de antibiótico associado a corticosteróide e curativo oclusivo. No pósoperatório, utilizou-se a associação de ofloxacina e prednisolona a $0,1 \%$, tópicos, uma gota de 6 em 6 horas, diminuindo-a gradativamente, até o desaparecimento dos sinais inflamatórios. As suturas de nylon 10-0 foram removidas sob biomicroscopia, com anestesia tópica, aproximadamente um mês após a cirurgia. Os pacientes foram seguidos por um período que variou de quatro a 26 meses (média de 14,0 \pm 7,9 meses).

Em razão do pequeno número de olhos estudados, os resultados serão apresentados na forma de relatos de casos, não tendo sido realizada análise estatística.

$\mathrm{Na}$ tabela 1, apresentam-se os dados demográficos dos pacientes e os achados nos cinco olhos hipotônicos antes da ressecção da bolsa hiperfuncionante.

Na tabela 2, mostram-se os achados nos cinco olhos após a ressecção da bolsa hiperfuncionante.

Para ilustrar os achados antes e depois da ressecção da bolsa hiperfuncionante, são mostradas as fotografias obtidas do paciente com maior tempo de seguimento (paciente 1).

\section{Paciente 1}

JAR, sexo masculino, 20 anos, leucodérmico, com glaucoma no olho esquerdo (OE), secundário a uveíte posterior por toxoplasmose. A gonioscopia desse olho mostrava goniossinéquias sectoriais. Foi submetido em outro Serviço a trabeculectomia com aplicação per-operatória de mitomicina $\mathrm{C}(0,33 \mathrm{mg} / \mathrm{ml}$, durante 3 minutos), no OE, em 22/03/2001, dois meses após o término do tratamento da uveíte. No pós-operatório, evoluiu com hipotonia no $\mathrm{OE}(\mathrm{Po}=0 \mathrm{mmHg}$ ), associada com bolsa elevada e avascular e descolamento de coróide visível à ecografia B. Chegou ao nosso Serviço com Po igual a $0 \mathrm{mmHg}$, sob atropina a 1,0\% colírio, uma gota duas vezes ao dia e dexametasona a $0,1 \%$, colírio, uma gota quatro vezes ao dia há cerca de trinta dias. À biomicroscopia convencional apresentava bolsa avascular elevada e difusa (Figura 1a), ausência de sinais inflamatórios no segmento anterior e teste de Seidel negativo. À UBM, não havia DCC. A oftalmoscopia mostrou edema macular, estrias e dobras na coróide no OE (Figura 1b). A acuidade visual desse olho era igual a 0,5 sob correção. Diante do quadro clínico apresentado e da ausência de resposta ao tratamento clínico, o paciente, após consentimento informado, foi submetido à ressecção da bolsa hiperfuncionante em 21/06/ 01, com subseqüente normalização da Po do OE. Esse paciente é o que apresenta maior tempo de seguimento. Na última visita, 
26 meses após a cirurgia, apresentava no OE, acuidade visual igual a 1, sem correção, Po igual a 14 mmHg, sem medicação; à biomicroscopia, apresentava bolsa pouco elevada e levemente anêmica (Figura 1c) e, ao exame oftalmoscópico, retina com aspecto normal (ausência de edema macular, de estrias e dobras na coróide) e escavação do disco óptico igual a 0,6 (Figura 1d).

\section{Paciente 2}

ABZB, masculino, 7 anos, feodérmico, portador de nistagmo e glaucoma congênito em ambos os olhos (AO), diagnosticado nos primeiros dias de vida. Foi submetido a trabeculotomia em AO aos 45 dias de vida. Reoperado do OD (TREC sem antimetabólito) com 5 meses de vida. Foi novamente reoperado de AO aos 2 anos de vida, em outro Serviço, trabeculectomia com MMC em 06/08/97 (não foi possível obter informações sobre a concentração e o tempo de uso da MMC). Retornou ao nosso Serviço um ano depois (24/08/98), quando foi diagnosticada hipotonia em $\mathrm{AO}(\mathrm{Po}=0 \mathrm{mmHg}$ no OD e igual a $2 \mathrm{mmHg}$ no OE). Foi medicado, em AO, com atropina a $0,5 \%$ colírio, uma gota duas vezes ao dia e dexametasona a $0,001 \%$, colírio, uma gota quatro vezes ao dia. Foi agendado reexame em uma semana, porém o paciente só retornou treze meses depois (24/09/99), quando apresentava Po igual a $0 \mathrm{mmHg}$ no OD e igual a $6 \mathrm{mmHg}$ no OE, sem medicação. Nessa ocasião, apresentava, à biomicroscopia do OD, dobras da membrana de Descemet, bolsa elevada e avascular com teste de Seidel negativo, opacificação cristaliniana polar posterior (possivelmente de origem cortisônica) e visão de percepção luminosa com percepção de cores. Foi novamente medicado no OD com atropina a $0,5 \%$ colírio, uma gota duas vezes ao dia e dexametasona a $0,1 \%$, colírio, uma gota quatro vezes ao dia. O exame oftalmoscópico foi inviável pela alterações corneanas e cristalinianas. Dois meses depois (23/11/99), a Po do OE era igual a $10 \mathrm{mmHg}$, sob medicação, porém a do OD permaneceu igual a zero. Durante cerca de dois anos, tentou-se reverter a hipotonia do OD substituindo-se a atropina a $0,5 \%$ colírio, uma gota duas vezes ao dia e a dexametasona a $0,1 \%$, colírio, pela atropina a $1 \%$ e prednisolona a $0,1 \%$, uma gota de cada colírio de $8 \mathrm{em} 8$ horas, porém a Po não ultrapassava $4 \mathrm{mmHg}$, valor este registrado respectivamente em 17/12/99 e 16/01/00. Em 12/

\begin{tabular}{|c|c|c|c|c|c|c|c|c|c|c|c|}
\hline $\begin{array}{l}\text { Caso/ } \\
\text { Olho }\end{array}$ & $\begin{array}{l}\text { Idade } \\
\text { (anos) }\end{array}$ & Sexo & Cor & Glaucoma & AV & Po & $\begin{array}{l}\text { N. de cir. } \\
\text { prévias }\end{array}$ & BF & $\begin{array}{c}\text { Alterações } \\
\text { de FO }\end{array}$ & $\begin{array}{c}\text { Teste de } \\
\text { Seidel }\end{array}$ & UBM \\
\hline $1 / O E$ & 20 & M & Leuco & $\begin{array}{l}\text { Sec. a } \\
\text { uveíte }\end{array}$ & 0,5 & zero & 1 & Avasc. & $\begin{array}{c}\text { Edema } \\
\text { macular, } \\
\text { estrias e dobras } \\
\text { coroidianas }\end{array}$ & Negativo & $\begin{array}{l}\text { Ausência } \\
\text { de DCC }\end{array}$ \\
\hline $2 / O D$ & 7 & M & Feo & Congênito & PL & zero & 3 & Avasc. & Inviável & Negativo & $\begin{array}{l}\text { Ausência } \\
\text { de DCC }\end{array}$ \\
\hline $3 / O D$ & 25 & M & Leuco & Congênito & PL & 2 & 4 & Avasc. & Inviável & Negativo & $\begin{array}{l}\text { Ausência } \\
\text { de DCC }\end{array}$ \\
\hline $4 / O E$ & 11 & M & Feo & Congênito & Vultos & 2 & 1 & Avasc. & $\begin{array}{l}\text { Mac. hipo- } \\
\text { tônica }\end{array}$ & Negativo & $\begin{array}{l}\text { Ausência } \\
\text { de DCC }\end{array}$ \\
\hline $5 / \mathrm{OE}$ & 50 & $\mathrm{~F}$ & Feo & $\begin{array}{c}\text { Sec. a } \\
\text { traumatismo }\end{array}$ & 0,2 & 3 & 1 & Avasc. & $\begin{array}{l}\text { Mac. hipo- } \\
\text { tônica }\end{array}$ & Negativo & $\begin{array}{l}\text { Ausência } \\
\text { de DCC }\end{array}$ \\
\hline
\end{tabular}

\begin{tabular}{|c|c|c|c|c|c|c|c|c|c|}
\hline $\begin{array}{l}\text { Caso/ } \\
\text { Olho }\end{array}$ & $\begin{array}{l}\text { Idade } \\
\text { (anos) }\end{array}$ & Sexo & Cor & Glaucoma & AV & Po $(\mathrm{mmHg})$ & BF & $\begin{array}{c}\text { Alterações } \\
\text { de FO }\end{array}$ & $\begin{array}{c}\text { Seguimento } \\
\text { (meses) }\end{array}$ \\
\hline $1 / \mathrm{OE}$ & 20 & M & Leuco & $\begin{array}{l}\text { Sec. a } \\
\text { uveíte }\end{array}$ & 1 & 14 & $\begin{array}{l}\text { Levemente } \\
\text { anêmica }\end{array}$ & Não & 26 \\
\hline 2/OD & 7 & $M$ & Feo & Congênito & 0,2 & 14 & $\begin{array}{l}\text { Levemente } \\
\text { anêmica }\end{array}$ & $C / D=0,8$ & 19 \\
\hline 3/OD & 25 & M & Leuco & Congênito & CD $0,5 \mathrm{~m}$ & 17 & $\begin{array}{l}\text { Levemente } \\
\text { anêmica }\end{array}$ & $\begin{array}{l}\text { Exame } \\
\text { Inviável }\end{array}$ & 14 \\
\hline $4 / \mathrm{OE}$ & 11 & $M$ & Feo & Congênito & 0,05 & 10 & $\begin{array}{l}\text { Levemente } \\
\text { anêmica }\end{array}$ & Não & 6 \\
\hline $5 / \mathrm{OE}$ & 50 & $\mathrm{~F}$ & Feo & $\begin{array}{c}\text { Sec. a } \\
\text { traumatismo }\end{array}$ & 0,3 & 10 & $\begin{array}{l}\text { Levemente } \\
\text { anêmica }\end{array}$ & Tênues & 5 \\
\hline
\end{tabular}



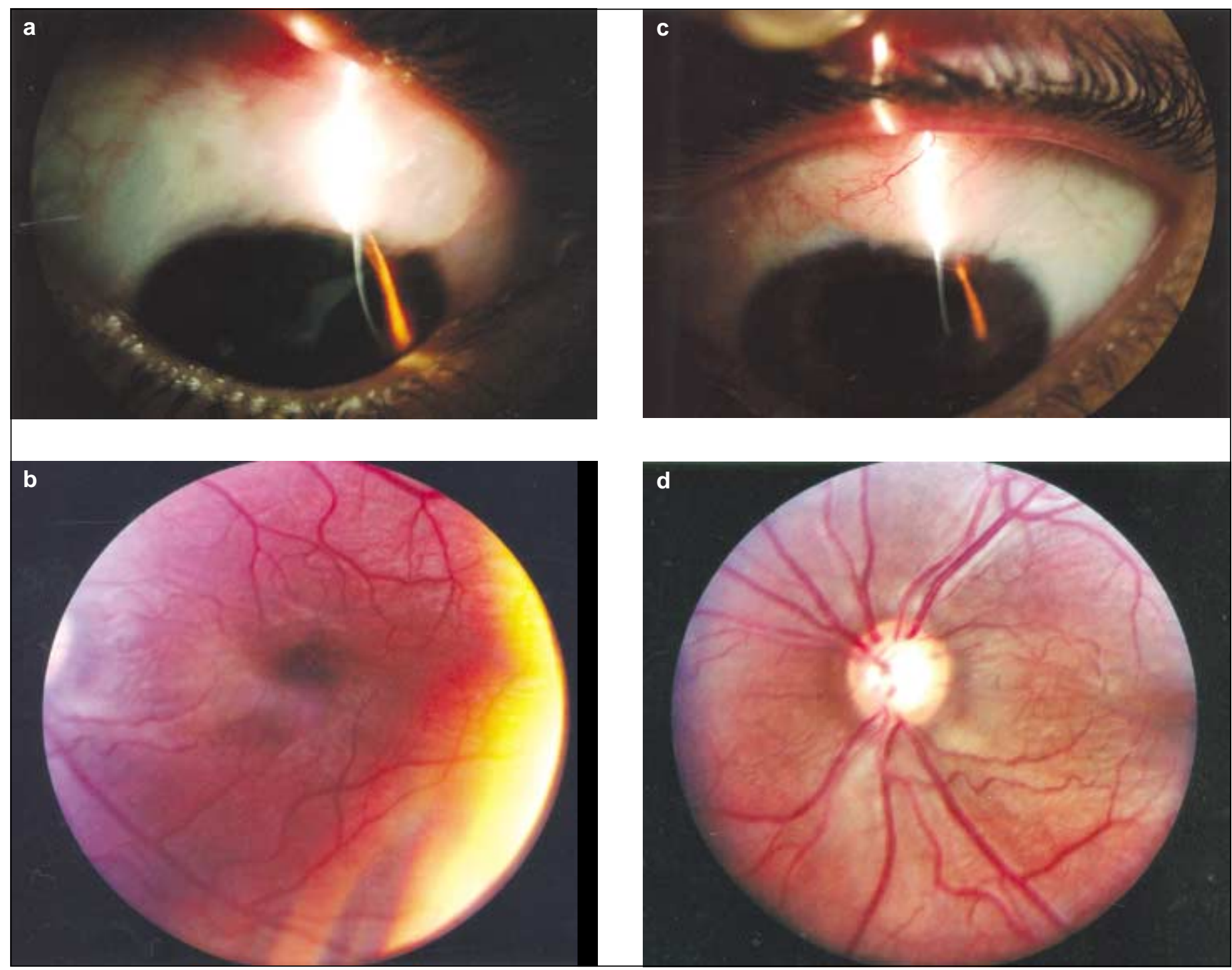

Figura 1 - Paciente 1: a) Fotografia mostrando bolsa avascular e difusa (hiperfuncionante); b) Retinografia mostrando edema macular, estrias e dobras na coróide; c) Aspecto da nova bolsa (pouco elevada e levemente anêmica) 26 meses após a resseccão da bolsa hiperfuncionante. Observar a conjuntiva cicatrizada na periferia da córnea; d) Retinografia mostrando a retina com aspecto normal e escavação do disco óptico ligeiramente aumentada $(0,6) 26$ meses após a ressecção da bolsa hiperfuncionante

09/90, o paciente apresentava à biomicroscopia do OD, dobras da membrana de Descemet, bolsa fistulante elevada e avascular e catarata total do tipo intumescente no OD. Foram realizados exames de ecografia B e de UBM que mostraram, respectivamente, que a retina encontrava-se aplicada e que não havia DCC no OD. Decidiu-se, então, submeter o paciente, sob consentimento informado dos seus pais, no mesmo ato operatório, à facectomia extra-capsular com implante de lente intraocular e à ressecção da bolsa hiperfuncionante, o que foi feito em 06/12/01. Passados 4 meses da cirurgia, foi submetido a capsulotomia com Nd:YAG laser. Teve boa evolução no pósoperatório, com a Po do OD igual a $14 \mathrm{mmHg}$, sem medicação e a visão igual a 0,2 , sob correção, 19 meses após a ressecção. À biomicroscopia, o OD apresentava bolsa pouco elevada e levemente anêmica com teste de Seidel negativo e, à oftalmos- copia, com a exceção da escavação glaucomatosa, não havia outras alterações.

\section{Paciente 3}

JBL, sexo masculino, 25 anos, leucodérmico, portador de glaucoma congênito descoberto aos seis meses de vida. Já havia sido operado quatro vezes em outro Serviço, a primeira cirurgia aos oito meses de vida. Na quarta cirurgia, realizada em março de 1996 em AO, foi utilizada MMC per-operatória (não foi possível obter informações sobre a concentração e o tempo de uso da MMC). Ao retornar ao nosso Serviço em 21/ 10/97, apresentava Po à aplanação igual a zero em AO; buftalmia bilateral; visão de percepção luminosa no OD e visão zero no OE. À biomicroscopia, apresentava no OD edema difuso e acentuado de córnea, bolsa elevada, avascular com microcis- 
tos no seu interior e com teste de Seidel negativo. O exame oftalmoscópico foi inviável pelo edema de córnea. Foi realizada uma ecografia que não mostrou descolamento coroidiano. Entretanto, após a realização da UBM, evidenciou-se pequeno DCC no quadrante temporal do OD. Tendo em vista tratar-se de olho único, buftálmico, já submetido a quatro cirurgias e observando-se a resistência do paciente a qualquer tipo de cirurgia (paciente reside em outro Estado), tentou-se reverter a hipotonia do OD durante cerca de quatro anos substituindose a dexametasona a $0,1 \%$, pela prednisolona a $0,1 \%$, uma gota de 6 em 6 horas, associando-a a atropina colírio a $1 \%$ uma gota de 12 em 12 horas, porém a Po não ultrapassou $2 \mathrm{mmHg}$.

Repetindo-se a UBM, observou-se o desaparecimento do DCC no quadrante temporal. Como a Po mantinha-se em zero, apesar da ausência de DCC à UBM nos quatro quadrantes e do teste de Seidel negativo, decidiu-se mais uma vez convencer o paciente da necessidade de tentar a cirurgia de ressecção da bolsa hiperfuncionante no OD, que foi realizada, sob consentimento informado do paciente, em 23/06/02. No per-operatório, curiosamente, observou-se laceração escleral linear de aproximadamente $3 \mathrm{~mm}$ de extensão, por onde havia drenagem copiosa de humor aquoso. A laceração foi suturada com Vycril $7-0^{\mathrm{TM}}$ e a bolsa hiperfuncionante ressecada. Quarenta e cinco dias após a ressecção, apresentava, à biomicroscopia, bolsa elevada e vascularizada e edema moderado da córnea.

Na última visita, 14 meses após a ressecção da bolsa, a Po do OD era igual a $17 \mathrm{mmHg}$, sem medicação e a visão que era de percepção luminosa passou a ser de conta dedos a 0,5 metro. À biomicroscopia, o OD apresentava bolsa pouco elevada e pouco vascularizada com teste de Seidel negativo e a córnea apresentava regressão acentuada do edema. O exame oftalmoscópico era ainda inviável.

\section{Paciente 4}

SSG, sexo masculino, 11 anos, feodérmico, nasceu com glaucoma congênito em ambos os olhos. Apresentava nistagmo. Foi submetido a trabeculotomia em ambos os olhos em 23/ 06/1992 aos doze dias de vida. Em 22/08/2000, aos oito anos de idade, foi submetido em AO, a TREC com MMC, $0,5 \mathrm{mg} / \mathrm{ml}$, durante cinco minutos, embebida em esponja de celulose e colocada sobre a esclera antes da demarcação do retalho escleral. Foi feita lavagem copiosa da esclera e dos fundos de sacos conjuntivais com $40 \mathrm{ml}$ de soro fisiológico a $0,9 \%$. O retalho escleral foi suturado com cinco pontos de Vycril 7- $0^{\mathrm{TM}}$, sendo dois pontos nos ângulos do retalho escleral, um ponto entre eles e um ponto de cada lado do retalho escleral. A conjuntiva foi fechada com sutura contínua utilizando-se também o Vycril 7-0 $0^{\mathrm{TM}}$. Uma semana após a cirurgia, apresentava Po igual a zero em AO, usando atropina colírio a 1\% de 12 em 12 horas e colírio de dexametasona a $0,1 \%$ de 6 em 6 horas. Um mês após a cirurgia a Po era igual a $6 \mathrm{mmHg}$ no OD e $3 \mathrm{mmHg}$ no OE. À biomicroscopia, apresentava em AO, bolsas elevadas e grandes, avasculares, com suspeita diagnóstica de serem hiperfuncionantes. Não havia sinais inflamatórios na câmara anterior. O teste de Seidel foi negativo. Em 28/09/00, realizou-se UBM em AO que não mostrou DCC. O paciente foi seguido de 10/10/00 a 28/10/02, período em que a Po variou de 4 a $6 \mathrm{mmHg}$ no OD e de 2 a $4 \mathrm{mmHg}$ no OE. Dentro deste período, repetiu-se a UBM em $\mathrm{AO}$ que mais uma vez não mostrou DCC. Durante todo o seguimento, a visão do OD permaneceu boa, porém no OE a visão reduziu-se a vultos em conseqüência de maculopatia hipotônica diagnosticada em 21/11/00. Em 28/02/03, a Po no OD era igual a $8 \mathrm{mmHg}$ no OD e $2 \mathrm{mmHg}$ no OE. Diante do quadro clínico apresentado e da ausência de resposta ao tratamento clínico, o paciente, após consentimento informado assinado por seu pai, foi submetido à ressecção da bolsa hiperfuncionante do $\mathrm{OE}$ na mesma data (28/02/03). Treze dias após a ressecção, a Po do OE era igual a $34 \mathrm{mmHg}$ sob atropina a $1 \%$, uma gota duas vezes ao dia e colírio de dexametasona a $0,1 \%$, uma gota de 6 em 6 horas. À biomicroscopia, o OE apresentava-se calmo, com bolsa moderadamente elevada e vascularizada. À oftalmoscopia, observou-se melhora do quadro de maculopatia hipotônica compatível com a melhora da acuidade visual de vultos para conta dedos a um metro, sem correção. Em face da Po muito elevada e da ausência de sinais inflamatórios, suspendeu-se a medicação do OE; o paciente retornou cinco dias depois, mas como a Po permanecia ainda elevada $(30 \mathrm{mmHg})$, prescreveu-se maleato de timolol a $0,5 \%$, uma gota de 12 em 12 horas. Uma semana depois, a Po do OE já era igual a $22 \mathrm{mmHg}$ sob timolol a $0,5 \%$ de 12 em 12 horas, associando-se a essa redução da Po uma melhora da visão (conta dedos a dois metros, sem correção). Dois meses depois, a Po do OE já era igual a $12 \mathrm{mmHg}$ sob timolol a $0,5 \%$ de 12 em 12 horas. Reduziu-se o timolol a $0,5 \%$ para uma gota pela manhã e um mês depois a Po do OE era igual a $10 \mathrm{mmHg}$. Suspendeu-se o timolol a $0,5 \%$ e no último retorno, seis meses após a cirurgia, a Po do OD era igual a $6 \mathrm{mmHg}$ e igual a $10 \mathrm{mmHg}$ no $\mathrm{OE}$, ambos os olhos sem medicação. À biomicroscopia, o OE apresentava bolsa moderadamente elevada e levemente anêmica. À oftalmoscopia, o OE apresentava-se com disco óptico com escavação fisiológica e mácula aparentemente normal. Neste olho, a visão melhorou de conta dedos para 20/400 sob correção de -4,75 esférico combinado com $-1,75$ cilindro, eixo a $175^{\circ}$.

\section{Paciente 5}

MRS, 50 anos, sexo feminino, feodérmica, em acompanhamento em nosso Serviço desde de 1995. No ano anterior, ao fazer exame com queixa de baixa acentuada da visão no OE foi feito o diagnóstico de glaucoma crônico simples. Não tinha queixas em relação ao OD. Naquela época, estava em uso de pilocarpina $2 \%$ duas vezes ao dia em $\mathrm{AO}$ e apresentava Po de $20 \mathrm{mmHg}$ no OD e $30 \mathrm{mmHg}$ do OE. A visão era 1,0 em sem correção, em ambos os olhos. A gonioscopia mostrava seio intermediário no OD, porém, no OE, o seio era muito amplo, com quadro típico de retrocesso da íris e do corpo ciliar em $360^{\circ}$, fazendo-se suspeitar de glaucoma traumático, embora a história de trauma nesse olho fosse negativa. A fundoscopia mostrava o nervo óptico com escavação igual a 0,3 no OD e 0,8 no OE. O campo visual do OD era normal, enquanto que o do 
OE já mostrava importante escotoma arqueado no hemicampo inferior.

Tentou-se o tratamento clínico inicialmente com betaxolol, pilocarpina e dipivalilepinefrina, todos duas vezes ao dia e apenas no OE, sem nenhum resultado, realizando-se, então, a primeira trabeculectomia neste olho, no quadrante nasal superior, em novembro de 1995. A Po estabilizou-se em $20 \mathrm{mmHg}$ nos meses seguintes, sem medicação, porém, menos de um ano depois, era igual a $30 \mathrm{mmHg}$, reiniciando-se, assim, o tratamento clínico. Novamente, frente à pobre resposta ao tratamento clínico, realizou-se outra trabeculectomia no $\mathrm{OE}$, em abril de 1997, desta vez no quadrante temporal superior utilizando-se a MMC na concentração de $0,5 \mathrm{mg} / \mathrm{ml}$, durante 5 minutos. Inicialmente, a Po estabilizou-se em $10 \mathrm{mmHg}$ sem medicação, porém, um ano depois, o OE começou a apresentar períodos de hipotonia, com a Po variando de 5 a $10 \mathrm{mmHg}$. $\mathrm{Na}$ região da TREC, havia-se formado grande bolsa, redundante sobre a córnea, difusa, muito elevada e avascular.

Em dezembro de 2002, a Po do OE era igual a $3 \mathrm{mmHg}$ e a acuidade visual reduziu-se para 0,2 sob correção. A fundoscopia mostrava nítidas dobras coriorretinianas no feixe papilomacular e na mácula, confirmando o diagnóstico de maculopatia hipotônica. O teste de Seidel mostrou-se negativo e a UBM não evidenciou DDC em nenhum dos quatro quadrantes, confirmando o diagnóstico de bolsa hiperfuncionante. Foi tentado o tratamento clínico da hipotonia com atropina colírio a $1 \%$ duas vezes ao dia e dexametasona colírio a $0,1 \%$ quatro vezes ao dia durante pelo menos trinta dias. Como não houve normalização da Po, em abril de 2003, a paciente foi, então, submetida, sob consentimento informado, à ressecção da bolsa hiperfuncionante. Com 15 dias de pós-operatório, a Po subiu para $15 \mathrm{mmHg}$, estabilizando-se em torno de 10 a $12 \mathrm{mmHg}$ a partir do primeiro mês de pós-operatório, quando a paciente já referia melhora importante da visão. Na última revisão, cinco meses após a cirurgia, o OE apresentava bolsa ligeiramente anêmica bem menor do que a apresentada antes da ressecção e a Po era igual a $11 \mathrm{mmHg}$ sem medicação. A acuidade visual do OE era igual a 0,3 sob correção; a oftalmoscopia mostrava discretas dobras coroidianas no feixe papilomacular, aparentemente não responsáveis pela não recuperação total da visão, atribuída ao surgimento de catarata subcapsular posterior diagnosticada à biomicroscopia.

\section{COMENTÁRIOS}

A hipotonia ocular pode ter consequiências dramáticas para a fisiologia neuro-retiniana, principalmente nos pacientes em que ela se estende por longo período ${ }^{(22)}$. Com a difusão do uso de antimitóticos na trabeculectomia, a hipotonia tornouse entidade relativamente frequiente no pós-operatório, exigindo tratamento adequado, pela potencial gravidade de suas complicações $^{(4)}$. A bolsa hiperfuncionante destaca-se como uma das causas de hipotonia ocular pós-trabeculectomia, sendo caracterizada por aspecto elevado, associado à ausência de vazamento de humor aquoso (teste de Seidel negativo) e de descolamento ciliocoroidiano à UBM.

O diagnóstico de hiperfunção é, na maioria das vezes, objeto de controvérsia. Na realidade, não há "hiperfiltração" na bolsa da TREC ou de qualquer cirurgia fistulante mas sim reabsorção excessiva de humor aquoso. Nos pacientes submetidos a TREC com antimitóticos, portadores de hipotonia ocular crônica, a hiperfunção, que é subclínica, só poderá ter o seu selo diagnóstico depois de excluírem-se as outras causas de hipotonia como o vazamento do humor aquoso pela bolsa (teste de Seidel positivo), presença de sinais inflamatórios na câmara anterior e descolamento ciliocoroidiano ao exame de biomicroscopia ultra-sônica.

É importante enfatizar que quatro dos cinco pacientes do presente trabalho eram jovens (idade variando de 7 a 25 anos), que, se de um lado, são sabidamente refratários à TREC sem antimetabólitos, do outro são os mais susceptíveis à instalação da hipotonia ocular e a outras complicações graves causadas pela MMC, destacando-se dentre elas, a maculopatia hipotônica, como ocorreu no paciente 4 (11 anos).

Numa série de nove pacientes ${ }^{(18)}$, foi relatada a presença de maculopatia hipotônica em cinco $(55,6 \%)$ olhos com hipotonia crônica pós-TREC primária com MMC. Os pacientes eram relativamente jovens, dois deles com idade de 18 anos, sendo a média de idade de 33,2 anos.

Portanto, há necessidade da busca de outras alternativas para o tratamento de glaucomas refratários em pacientes jovens.

Ao utilizar-se a MMC, que se o faça em concentrações mínimas e por menor tempo de aplicação e que o acompanhamento dos pacientes seja frequiente a fim de surpreender-se em tempo hábil às eventuais complicações.

Em contraste com os dados do presente trabalho, dois dos pacientes relatados na literatura nacional tinham idade de $92 \mathrm{e}$ 68 anos respectivamente ${ }^{(14,17)}$. Além disso, nos três trabalhos nacionais $^{(14,17-18)}$, não ficou claramente definido como foi feito o diagnóstico de hiperfunção ("hiperfiltração") da bolsa, nos moldes como aqui estabelecidos. No primeiro trabalho ${ }^{(14)}$, um paciente de 92 anos, com diagnóstico de bolha hiperfuncionante cística, com teste de Seidel negativo, apresentava visão de 20/200 e Po pré-operatória de $8 \mathrm{mmHg}$, não caracterizando bem uma hipotonia ocular. Neste paciente, os autores ${ }^{(14)}$ não relatam a realização de UBM. Ele foi submetido a um transplante de esclera de cadáver com colocação de membrana amniótica sobre o transplante, evoluindo com Po de $18 \mathrm{mmHg}$ sob medicação antiglaucomatosa no seguimento de 2 meses, a visão permanecendo inalterada. No segundo trabalho, os autores $^{(17)}$ não reportam à realização do teste de Seidel nem de UBM, no paciente de 68 anos. O paciente foi submetido à sutura de compressão da bolsa associada à cirurgia de catarata e a Po que era igual a $2 \mathrm{mmHg}$ no pré-operatório elevou-se para $10 \mathrm{mmHg}$, permanecendo a visão inalterada num período de seguimento de 40 dias. No terceiro trabalho, os autores ${ }^{(18)}$ relataram que nenhum dos nove olhos por eles estudados apresentou teste de Seidel positivo, o que lhes induziram a 
pensar ser a hiperfunção ("hiperfiltração") a base da hipotonia.

Várias formas de tratamento de hipotonia ocular pós-trabeculectomia têm sido descritas. O uso de curativo compressivo, lente de contato escleral ou hipotensores oculares que diminuam a produção de humor aquoso, reduzindo, pois a drenagem $^{(7)}$, não tinham indicação nos pacientes aqui estudados, já que todos apresentavam teste de Seidel negativo e ausência de DCC, além de não terem sido abordados no pós-operatório imediato (o mais precoce deles, três meses após a trabeculectomia). A injeção de sangue autólogo na bolsa tem sido descrita, com eficácia entre 60 e $75 \%$, mas reveste-se do alto risco de hifema. ${ }^{(6-10,18,26)}$ Através de mecanismo semelhante, tem sido proposto ${ }^{(16)}$. Ainda com esse laser, tem sido descrito o remodelamento da bolsa ${ }^{(15)}$.

A despeito da pequena casuística, este trabalho demonstra que a ressecção da bolsa hiperfuncionante associada à ancoragem da conjuntiva sadia à córnea, proporciona resultados muito satisfatórios. As outras opções de tratamento: sutura de compressão ${ }^{(17)}$, avanço da conjuntiva com preservação da bolsa ${ }^{(25)}$, enxerto conjuntival ${ }^{(11-12)}$, de fáscia lata ${ }^{(13)} \mathrm{ou}$ membrana amniótica ${ }^{(14)}$ apresentaram resultados nem sempre satisfatórios como os aqui apresentados. A nosso ver, isto pode ser devido a fatores inerentes a cada técnica e, principalmente, à falha no diagnóstico correto da causa da hipotonia ocular. Assim sendo, o diagnóstico correto da causa da hipotonia é fundamental para a decisão da melhor conduta a ser tomada para lograr-se a sua reversão.

É necessário enfatizar que a hipotonia pode ser devida a bolsa hiperfuncionante, vazamento de humor aquoso pela bolsa (teste de Seidel positivo), reação inflamatória na câmara anterior e descolamento ciliocoroidiano primário. Havendo vazamento de humor aquoso pela bolsa (teste de Seidel positivo), a nossa conduta não se restringe apenas à ressecção da bolsa mas também à colocação de um enxerto de esclera no local da bolsa para proteger a nova bolsa a ser formada e evitar a recidiva do vazamento. Na vigência de reação inflamatória intensa na câmara anterior, o tratamento deve ser feito com colírio cicloplégico (por exemplo, atropina a $1 \%$, uma gota de oito em oito horas) associado ao uso de corticosteróide tópico (dexametasona ou betametasona a $0,1 \%$ ou prednisolona a $1 \%$, uma gota de seis em seis horas) ${ }^{(25)} \mathrm{e}$, às vezes, sistêmico. Se houver um DCC primário, a conduta cirúrgica (na falha do tratamento clínico convencional) é a drenagem do fluido supracoroidiano associada à injeção intracamerular de substância viscoelástica e/ou a sutura do corpo ciliar.

Diante de uma bolsa hiperfuncionante, freqüentemente fragilizada pelo efeito dos antimetabólitos, a nossa conduta tem sido a sua ressecção que, além de permitir a reconstituição protetora da região onde ela se encontrava, permite a exploração per-operatória da esclera e trajeto fistulante subjacentes. Em um dos nossos pacientes, essa exploração revelou uma laceração escleral, radial, não evidenciada à UBM, através da qual havia drenagem copiosa de humor aquoso. Tratava-se de um paciente portador de glaucoma congênito (paciente 3), submetido a quatro cirurgias fistulantes em outro Serviço, tendo sido utilizada MMC per-operatória na última cirurgia.

A ressecção da bolsa hiperfuncionante tem também a vantagem de eliminar a conjuntiva papirácea e reconstituir a bolsa com tecido potencialmente sadio, adjacente a ela, diferentemente do que ocorre ao utilizar-se a técnica de avanço da conjuntiva com preservação da bolsa ${ }^{(25)}$. Três dos cinco pacientes aqui estudados eram provenientes de outros Serviços, dois deles, operados em outro Estado. Três eram portadores de glaucoma congênito, um deles operado duas vezes (paciente 4), outro (paciente 2), três vezes e, o outro quatro vezes (paciente 3), em todos eles tendo sido feito uso per-operatório de MMC na última cirurgia. O último paciente (50 anos) era portador de glaucoma secundário a traumatismo.

É importante realçar a melhora da acuidade visual que ocorreu nos cinco pacientes, tendo sido possível registrar a reversão das alterações oftalmoscópicas causadas pela hipotonia nos pacientes 1, 4 e 5 (Figura 1d). É necessário encarecer que a reversão das alterações corio-retinianas (dobras de coróide e maculopatia) causadas pela hipotonia ocular dependerá do diagnóstico correto e do tratamento adequado da sua causa, como ocorreu prontamente nos pacientes 1 e 5 (respectivamente três e cinco meses após o diagnóstico). Por outro lado, no paciente 4 , com seguimento de seis meses, no qual a maculopatia hipotônica foi diagnosticada em 21/11/00 e a ressecção da bolsa hiperfuncionante com normalização da Po só ocorreu mais de dois anos e três meses depois, a visão do $\mathrm{OE}$ não retornou aos níveis normais, a despeito da aparente reversão da maculopatia hipotônica em decorrência do nistagmo e ambliopia. Portanto, nos três pacientes, portadores de glaucoma congênito, não houve recuperação completa da visão, o que pode ser atribuído não somente às alterações funcionais (nistagmo, ambliopia, etc) decorrentes da refratariedade do glaucoma congênito mas também à demora, por motivos variados, na realização do procedimento para reversão da hipotonia ocular (mais de três anos no paciente 2, mais de quatro anos no paciente 3 e dois anos e três meses no paciente 4). Diferentemente do que ocorre na progressão do glaucoma crônico simples, na hipotonia ocular, as alterações retinianas, em geral, se instalam de maneira rápida, sendo tanto mais graves quanto mais tempo e mais baixa se mantém a Po, o que reduzirá também as chances de sucesso do tratamento clínico e/ou cirúrgico.

No presente trabalho, a ressecção da bolsa hiperfuncionante mostrou-se eficaz no tratamento da hipotonia ocular crônica, sendo um procedimento de execução relativamente simples e morbidade reduzida, além de permitir a exploração per-operatória do leito escleral, que pode estar acometido em pacientes submetidos a várias reoperações com o uso de antimetabólitos.

\section{ABSTRACT}

To present the results of bleb resection for the treatment of 
overfiltering bleb after trabeculectomy with mitomycin $\mathrm{C}$ (MMC) associated with chronic ocular hypotony. Five patients with chronic ocular hypotony caused by overfiltering bleb underwent bleb resection. The authors established the criteria for the diagnosis of overfiltering bleb. Ocular hypotony was reversed in all patients without medication. The mean follow-up was $14.0 \pm 7.9$ months. Bleb resection is a good approach for the treatment of chronic ocular hypotony secondary to overfiltering bleb.

Keywords: Ocular hypotension; Trabeculectomy; Glaucoma; Mitomycin/therapeutic use; Injections; Intraocular pressure; Postoperative complications; Treatment outcome; Case report

\section{REFERÊNCIAS}

1. Jay JL, Murdoch JR. The rate of visual field loss in untreated primary open angle glaucoma. Br J Ophthalmol. 1993;77(3):176-8.

2. Migdal C, Gregory W, Hitchings R. Long term functional outcome after early surgery compared with laser and medicine in open angle glaucoma. Ophthalmology. 1994;101(10):1651-7.

3. Collaborative Normal-Tension Glaucoma Study Group. The effectiveness of intraocular pressure reduction in the treatment of normal tension glaucoma. Am J Ophthalmol. 1998;126(4):498-505.

4. Shigeeda T, Tomidokoro A, Araie M, Koseki N, Yamamoto S. Long-term follow-up of visual field progression after trabeculectomy in progressive normal-tension glaucoma. Ophthalmology. 2002;109(4):766-70.

5. Khaw PT, Wilkins M. Antifibrotic agents in glaucoma surgery. In: Yanoff M, Duker J. Ophthalmology. London: Mosby; 1999. p. 1-7.

6. Wise JB. Treatment of chronic postfiltration hypotony by intrableb injection of autologous blood. Arch Ophthalmol. 1993;111(6):827-30.

7. Leen MM, Takahashi Y, Li Y, Stewart JF, Chen PP, Mills RP. Mitotic effect of autologous blood injection and diode laser bleb revision on rabbit filtration blebs. Arch Ophthalmol. 1999;117(1):77-83.

8. Leen MM, Moster MR, Katz LJ, Terebuh AK, Schmidt CM, Spaeth GL. Management of overfiltering and leaking blebs with autologous blood injection. Arch Ophthalmol. 1995;113(8):1050-5.

9. Meyer GC, Meyer RC, Vilela M, Ferreira P, Domenico MD. Injeção "intrableb" de sangue autólogo no tratamento da hipotonia persistente. Rev Bras Oftalmol. 1996;55(9):669-72.
10. Choudhri AS, Herndon LW, Damji KF, Allingham RR, Shields MB. Efficacy of autologous blood injection for treating overfiltration or leaking blebs after glaucoma surgery. Am J Ophthalmol. 1997;123(4):554-5.

11. Buxton JN, Lavery KT, Liebmann JM, Buxton DF, Ritch R. Reconstruction of filtering blebs with free conjunctival autografts. Ophthalmology. 1994;101 (4):635-9.

12. Wilson RM, Kotas-Neumann R. Free conjunctival patch for repair of persistent late bleb leak. Am J Ophthalmol. 1994;117(5):569-74.

13. Hughes BA, Shin DH, Birt CM. Use of fascia lata in revision of filtration surgery. J Glaucoma. 1996;5(3):207-9.

14. Gomes JAP, Fernandes LHS, Komagome CM, Hofling-Lima AL, Prata-Jr. JA. Uso de membrana amniótica no tratamento de complicações pós-trabeculectomia. Arq Bras Oftalmol. 2001;64(5):437-41.

15. Lynch MG, Roesch M, Brown RH. Remodeling filtering blebs with the Nd:YAG laser. Ophthalmology. 1996;103(10):1700-5.

16. Bettin P, Carassa RG, Fiori M, Brancatto R. Treatment of hyperfiltering blebs with Nd:YAG laser-induced subconjunctival bleeding. J Glaucoma. 1999;8(6):380-3.

17. Ventura AGGM, Cavalcanti HDO, Holanda AGS, Cardoso G, Trigueiro L. Tratamento de bolhas hiperfiltrantes por sutura de compressão. Rev Bras Oftalmol. 2002;61(7):525-8.

18. Guedes VRF, Simmons RB, Pakter HM, Simmons RJ. Persistent hypotony after primary trabeculectomy with mitomycin C. Arq Bras Oftalmol. 2000;63 (3):179-83.

19. Fitzgerald JR, McCarthy JL. Surgery of the filtering bleb. Arch Ophthalmol. 1962;68(4):453-67.

20. Sugar S. Complications, repair and reoperation of antiglaucoma filtering blebs. Am J Ophthalmol. 1967;63(4):823-33.

21. Cohen JS, Shaffer RN, Hetherington Jr J, Hoskins D. Revision of filtration surgery. Arch Ophthalmol. 1977;95(9):1612-5.

22. Grostern RJ, Torczynski E, Brown SVL. Surgical repair and histopathologic features of a dissecting glaucoma filtration bleb. Arch Ophthalmol. 1999;117 (11):1566-7.

23. La Borwit SE, Quigley HA, Jampel HD. Bleb reduction and bleb repair after trabeculectomy. Ophthalmology 2000;107(4):712-8.

24. Catoira Y, Wudunn D, Cantor LB. Revision of disfunctional filtering blebs by conjunctival advancement with bleb preservation. Am J Ophthalmol, 2000; 130(5):574-9.

25. Becker B. Intraocular pressure response to topical corticosteroids. Invest Ophthalmol. 1965;26:198-205.

26. Haynes WN, Alward LM. Combination of autologous blood injection and bleb compression sutures to treat hypotony maculopathy. J Glaucoma. 1999;8 (6):384-8.

\section{CONGRESSO DA SOCIEDADE DE OFTALMOLOGIA DO TRIÂNGULO MINEIRO}

\section{8 a 20 de novernito de 2004 UBERLÂNDIA - MG}

INFO RMAÇÕES:

E-mail: interld@netsite.com.br 